Corrigendum

\title{
Corrigendum to "Tanshinone IIA Alleviates CCL2-Induced Leaning memory and Cognition Impairment in Rats: A Potential Therapeutic Approach for HIV-Associated Neurocognitive Disorder"
}

\author{
Yuan-jun Liao, ${ }^{1}$ Jian-min Chen, ${ }^{1}$ Jiang-yi Long, ${ }^{1}$ Yi-jun Zhou, ${ }^{1}$ Bing-yu Liang, \\ and Yan Zhou $\mathbb{D}^{1}$ \\ ${ }^{1}$ Department of Pharmacology, Guangxi Medical University, Nanning, Guangxi 530021, China \\ ${ }^{2}$ Guangxi Key Laboratory of AIDS Prevention and Treatment, Guangxi Medical University, Nanning, Guangxi 530021, China \\ Correspondence should be addressed to Yan Zhou; zhouyan@stu.gxmu.edu.cn
}

Received 14 December 2020; Accepted 14 December 2020; Published 24 December 2020

Copyright (c) 2020 Yuan-jun Liao et al. This is an open access article distributed under the Creative Commons Attribution License, which permits unrestricted use, distribution, and reproduction in any medium, provided the original work is properly cited.

Following the publication of the article "Tanshinone IIA Alleviates CCL2-Induced Leaning memory and Cognition Impairment in Rats: A Potential Therapeutic Approach for HIV-Associated Neurocognitive Disorder" [1], the authors identified that the incorrect images were presented in Figures $8(\mathrm{~d})$ and $8(\mathrm{~g})$. Figure $8(\mathrm{~g})$ was mistakenly duplicated with Figure 8(f), and Figure 8(d) was incorrectly taken from a different experiment. The authors explained that the error occurred during manuscript preparation and apologize for any inconvenience caused. The correct figure is as follows 


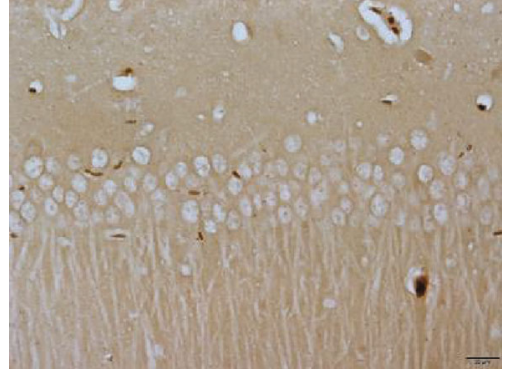

(a)

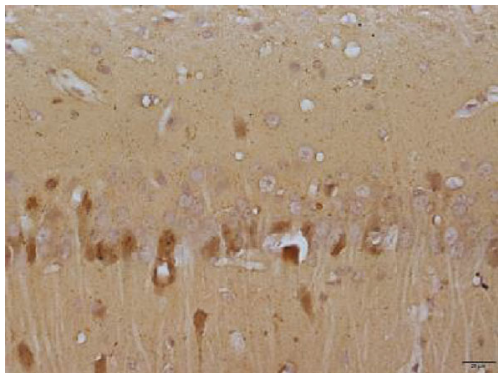

(c)

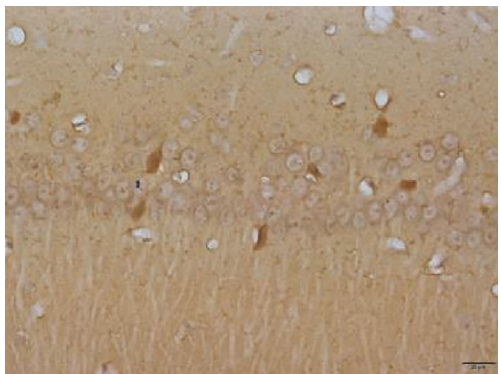

(e)

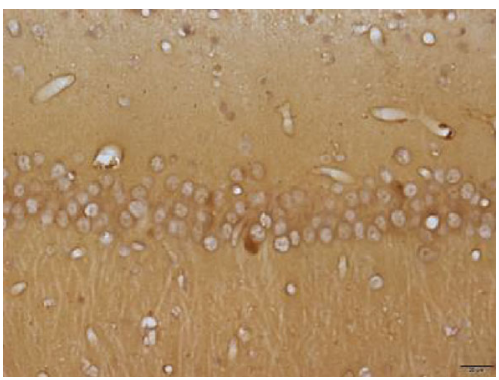

(g)

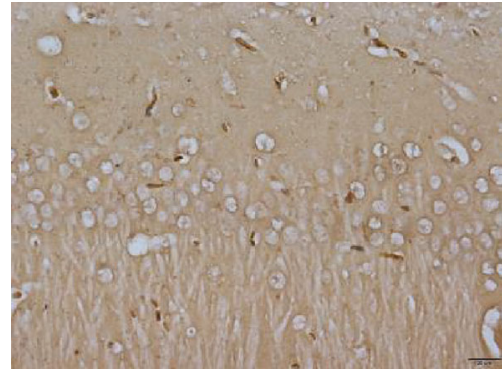

(b)

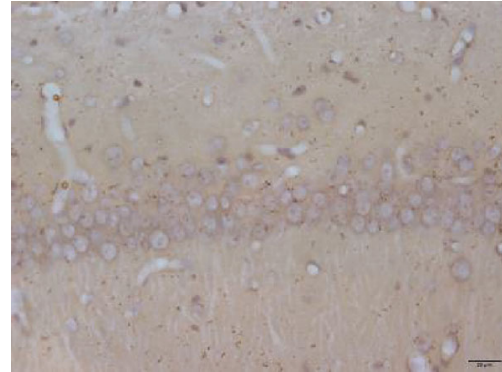

(d)

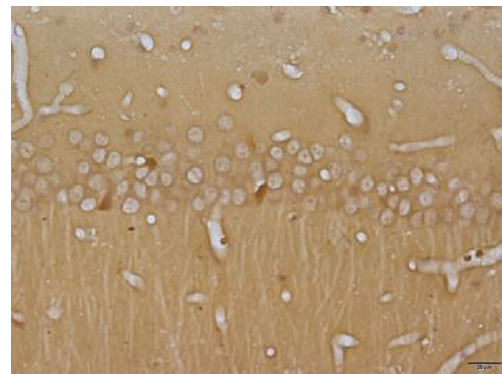

(f)

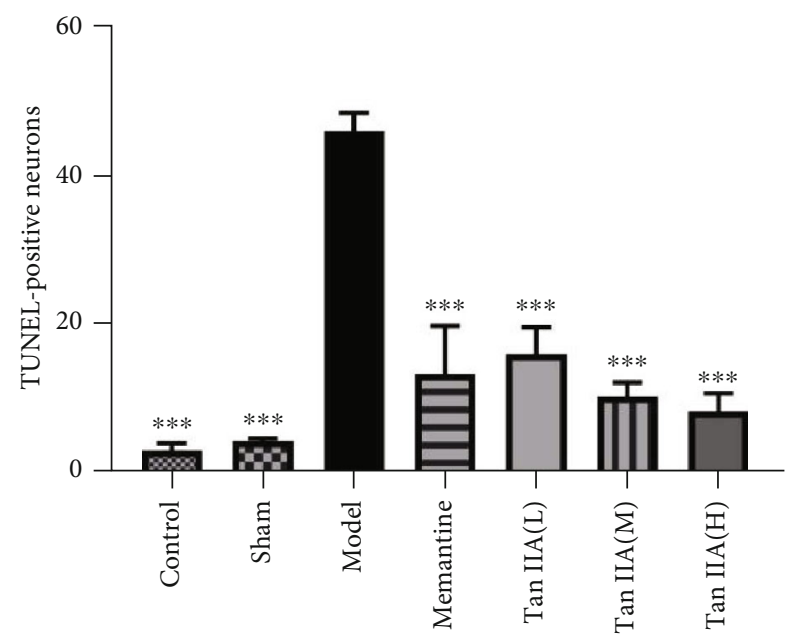

(h)

FIGURE 8: Cell apoptosis of hippocampal tissues in rats among the seven groups. (a-g) Representative 400× images of the TUNEL staining. TUNEL staining showed that Tan IIA reduced CCL2-induced cell apoptosis in the hippocampus CA1 areas, and the brown nuclei represented TUNEL-positive cells. (h) Cell apoptosis rate among seven groups. The results are expressed as mean $\pm \mathrm{SEM}, n=3 .{ }^{* * *} p<0.001$ versus the model group (Tan IIA(L): 25 mg/kg, Tan IIA(M): 50 mg/kg; Tan IIA(H): 75 mg/kg). (a) Control. (b) Sham. (c) Model. (d) Memantine. (e) Tan IIA(L). (f) Tan IIA(M). (g) Tan IIA(H). 


\section{References}

[1] Y.-j. Liao, J.-m. Chen, J.-y. Long, Y.-j. Zhou, B.-y. Liang, and Y. Zhou, "Tanshinone IIA Alleviates CCL2-Induced Leaning memory and Cognition Impairment in Rats: A Potential Therapeutic Approach for HIV-Associated Neurocognitive Disorder," BioMed Research International, vol. 2020, Article ID 2702175, 15 pages, 2020. 